\title{
Does mouse mammary tumor-like virus cause human breast cancer? Applying Bradford Hill criteria postulates
}

\author{
Yasir Hameed", Muhammad Usman and Mukhtiar Ahmad
}

\begin{abstract}
Background: The role of mouse mammary tumor-like virus (MMTV-like virus) in human breast cancer (BC) has already been widely investigated worldwide with conflicting results. Although the researchers tried to establish the link between MMTV-like virus and BC through the statistical meta-analysis of the previous studies associating MMTVlike virus with $B C$, they failed to establish a more reliable link due to the shortcomings of the statistical meta-analysis. In the present study, we identified population-wide studies relating MMTV-like virus with BC through the PubMed search engine. Then, we examined the available data of MMTV-like virus prevalence in BC, normal/benign samples, and applied the postulates of Bradford Hill criteria on the available evidence to investigate the association between MMTV-like virus and BC. In addition, to further enhance the reliability of our outcomes, we have also evaluated the methodologies of the previous studies to address the possibility of false-negative and false-positive results.

Results: After a careful evaluation of the extracted data against the postulates of Bradford Hill criteria, it was observed that none of the studies fulfill all the major postulates of Bradford Hill criteria for causation including temporality, consistency, biological gradient, experiment, coherence, specificity, and analogy. Hence, no causal relationship has been suggested between MMTV-like virus and BC patients of the any included population.
\end{abstract}

Conclusion: The results failed to prove the causal relationship between MMTV-like virus and BC rather suggested it as a co-participant in the pathogenesis of BC.

Keywords: Mouse mammary tumor-like virus (MMTV-like virus), Breast cancer (BC), Bradford Hill criteria

\section{Background}

Breast cancer $(\mathrm{BC})$ is a condition in which the breast cells grow abnormally and out of control. A breast is composed of three main parts: lobules, ducts, and connective tissue. The lobules are the glands that produce milk, while ducts are used to carry the milk to the nipple, and connective tissue surrounds and holds everything together (ZuccaMatthes et al. 2016). BC may occur at multiple sites in the breast but ducts and lobules are the major hotspots of the $\mathrm{BC}$. Based on the type of breast cells that turn into cancer, $\mathrm{BC}$ has been divided into several types (Sharma et al.

*Correspondence: Yasirhameed2011@gmail.com

Department of Biochemistry and Biotechnology, The Islamia University of Bahawalpur, Bahawalpur, Pakistan
2010). BC is one of the leading causes of mortality worldwide accounting for $14 \%$ of cancer-related deaths (Jemal et al. 2011) with a 5-year survival rate of approximately 40-80\% (Coleman et al. (2008)).

Being female, getting older, consuming alcohol, no pregnancy, obesity, and viral infection are the very common causes of the BC (Lawson 2009). Moreover, the role of human papilloma (HPV) and Epstein-Barr virus (EBV) in the development of $\mathrm{BC}$ has already been well acknowledged worldwide (Glenn et al. 2012). There is, however, no clear etiological role of mouse mammary tumor viruses (MMTV) have been identified in the development of $\mathrm{BC}$ since the first study which initially identified the presence of MMTV in human BC in 1972 (Axel et al. 1972; Oskouee et al. 2014). 
Another virus named MMTV-like virus is 95\% homologous to MMTV in terms of sequence homology (Lawson et al. 2006). Controversial evidence has been reported until now regarding the etiologic role of MMTV-like virus in the development of BC. Although the role of MMTV as an etiologic factor in the development of mice memory tumor is well established, however, it is still unclear whether MMTV-like virus contributes to the development of human BC.

The first-ever study documenting the presence of MMTV-like virus was conducted in 1972 by Axel et al. (1972) in the USA. In that study, they detected the presence of MMTV-like virus in a total of 38 BC samples using the molecular hybridization technique and results revealed the presence of MMTV-like virus in approximately $78.9 \%$ of the BC samples.

Since then, numerous studies have been carried out worldwide for detecting MMTV-like virus in BC and their outcomes were contradictory because MMTV-like virus was detected in varying detection positivity ratios population-wide, i.e., from 0\% (Oskouee et al. (2014); Bindra et al. 2007; Morales-Sanchez et al. 2013; Motamedifar et al. 2012; Park et al. 2011; Tabriz et al. 2013; Witt et al. 2003) to 100\% 78.9\% (Axel et al. 1972).

In general, a statistical meta-analysis is usually preferred when establishing a correlation between the virus and the disease as compared to the single study. This choice is based on the multiple advantages of the metaanalysis such as increased number of objects, greater diversity among the objects, and conclusion with a high level of evidence over the individual single study which has disadvantages like a small cohort of patients and conclusions with a low level of evidence. By keeping in view the inconsistencies in the MMTV-like virus detection ratios in worldwide published studies, recently, researchers have analyzed the previously published studies by the means of statistical meta-analysis to yield more useful pieces of information.

Previously, a statistical meta-analysis was performed to find out the causal relationship between MMTV-like virus and BC by Wang et al. (2014) through the available literature of MMTV-like virus and BC in various authentic research engines including MEDLINE (PubMed), Embase, EBSCO (ASP/BSP), and China National Knowledge Infrastructure (CNKI). They obtained more than 22 studies from different populations such as Western, Asian population and African populations and their revealed no significant association between MMTV-like virus and $\mathrm{BC}$.

Although evaluating the results of previous studies documenting the role of MMTV-like virus in the development of BC through statistical meta-analysis was a better choice than generalizing the results of an individual study, however, we did not consider statistical meta-analysis reliable to establish a causal-relationship between MMTV-like virus and the $\mathrm{BC}$ development because of some serious limitations such as its inability to analyze the methodologies of the previous studies, so there is no way to evaluate the possibility of false-negative and false-positive results, nor does statistical meta-analysis provide any information regarding the effect of heterogeneity-specific nature of the understudied populations on MMTV-like virus detection. In addition, statistical metaanalysis results in publication biasness, where meta-analysis does not select studies with no results even though they contain valuable information.

By looking at the discrepancy in the outcomes of the previously published studies and significant shortcomings of the statistical meta-analysis, we performed the population-wise valuation of the results of previous studies detecting MMTV-like virus in BC using the Bradford Hill criteria. These criteria are widely used and accepted worldwide over many years for establishing a causal relationship between a presumed cause and an observed effect of public health research (Fedak et al. 2015).

In the course of evaluation, we analyzed, whether or not these studies fulfill all the postulates of Bradford Hill criteria to declare a causal relationship between MMTVlike virus and $\mathrm{BC}$. In addition, we also evaluated the methodologies used by the previous studies to address the possibility of false-positive and false-negative results for better outcomes. The outcomes of the present study will help to establish a more reliable population-wise causal relationship between MMTV-like virus and BC and determine the more appropriate treatment strategies for $\mathrm{BC}$ patients.

\section{Methods}

In the present study, we implemented a two-phase methodology (Fig. 1).

\section{Literature search}

All the relevant articles associating MMTV-like virus with $\mathrm{BC}$ were identified through the PubMed search database using the keywords: "Breast Cancer" AND "MMTV-like virus." We also defined "Retroviridae" AND "Breast neoplasia" as medical subject headings (MeSH) terms. Mesh terms and keywords were combined during the search process. All the literature was searched available until March 2020, with the "Original Article" filter. In total, 1428 original articles were identified through the PubMed search engine.

\section{Relevant data extraction}

From 1429 original articles, the 40 relevant articles were identified having the desired information by initially 


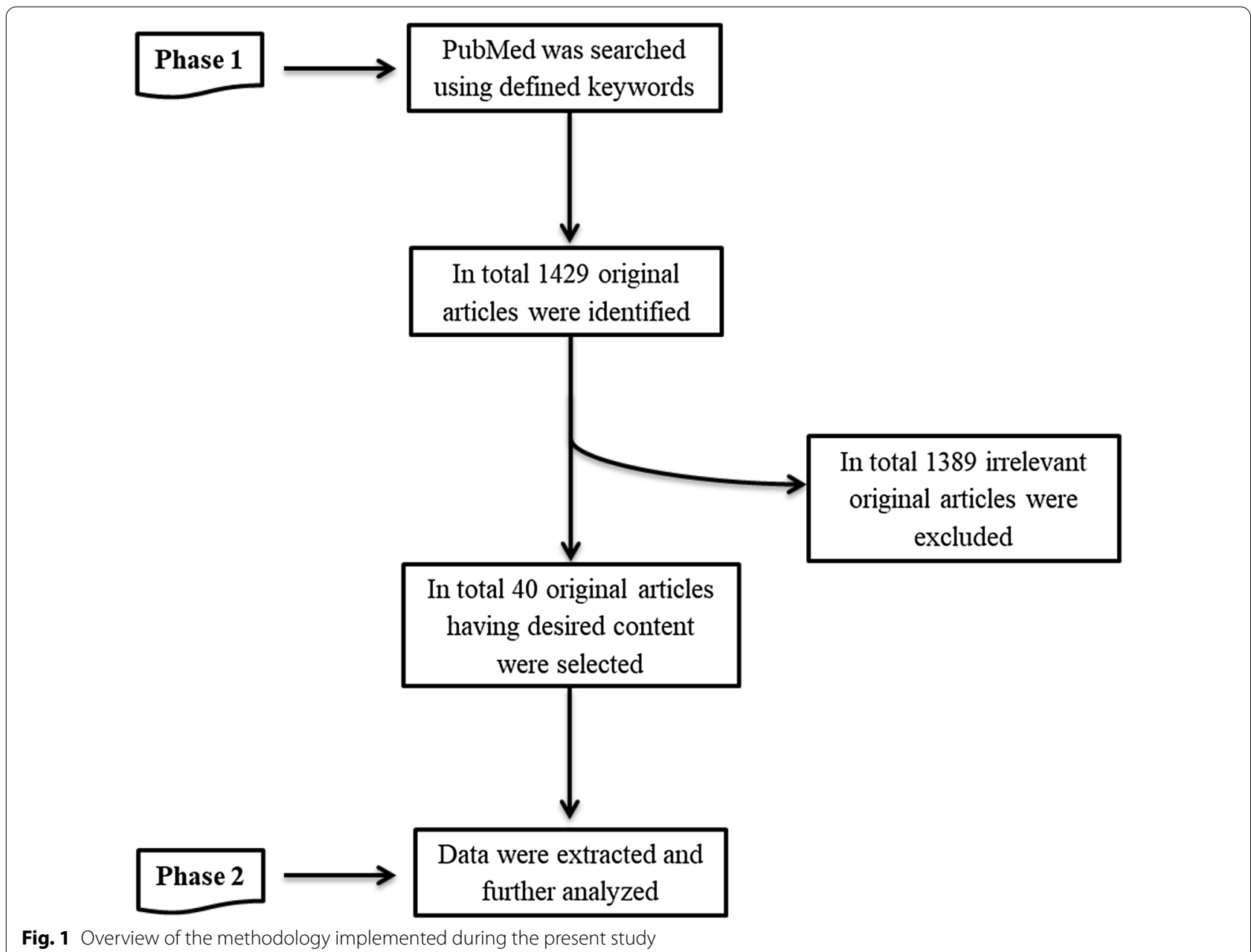

reading the title, abstract, and then the complete article. Furthermore, a comprehensive table was constructed having all the required information from the selected relevant studies.

\section{Evaluation of the results using the postulates of Bradford Hill criteria}

Based on the extracted data, all the identified studies were carefully evaluated against the following Bradford Hill criteria postulates:

(1) Strength: larger the association, more probability of the causal relationship, (2) Temporality: cause must lead to the induction of an effect. If the delay is expected between the cause and effect, then the effect has to occur after the delay, (3) Consistency: different studies conducted by different researchers at different places with different sample sizes and reporting the similar results increase the chances of the causal relation between the cause and effect, (4) Plausibility: there should be plausible mechanism between the cause and effect, (5) Biological gradient: greater response is produced by the causative agent in response to the greater exposure. However, in some cases, effect can be triggered by the mere presence of the factor, while in other cases, greater exposure can lead lower effect as well, (6) Experiment: the relationship between the cause and effect should be explained by the experiments and experiment should results in the reduction of effect when the causative agent is removed, (7) Coherence: causal relationship should not conflict with already known literature about the disease or exposure, (8) Specificity: causality is more likely if the effect has only one cause, (9) Analogy: previous evidence of the association between the cause and effect should support the current statement for the causal relationship.

The assessment of each postulate was qualitative/ descriptive, as there was an element of subjectivity in applying quantitative scoring. Evidence collected for each postulate is presented in Table 1 and results section with a final judgment as to whether the viewpoint was fulfilled or not. 


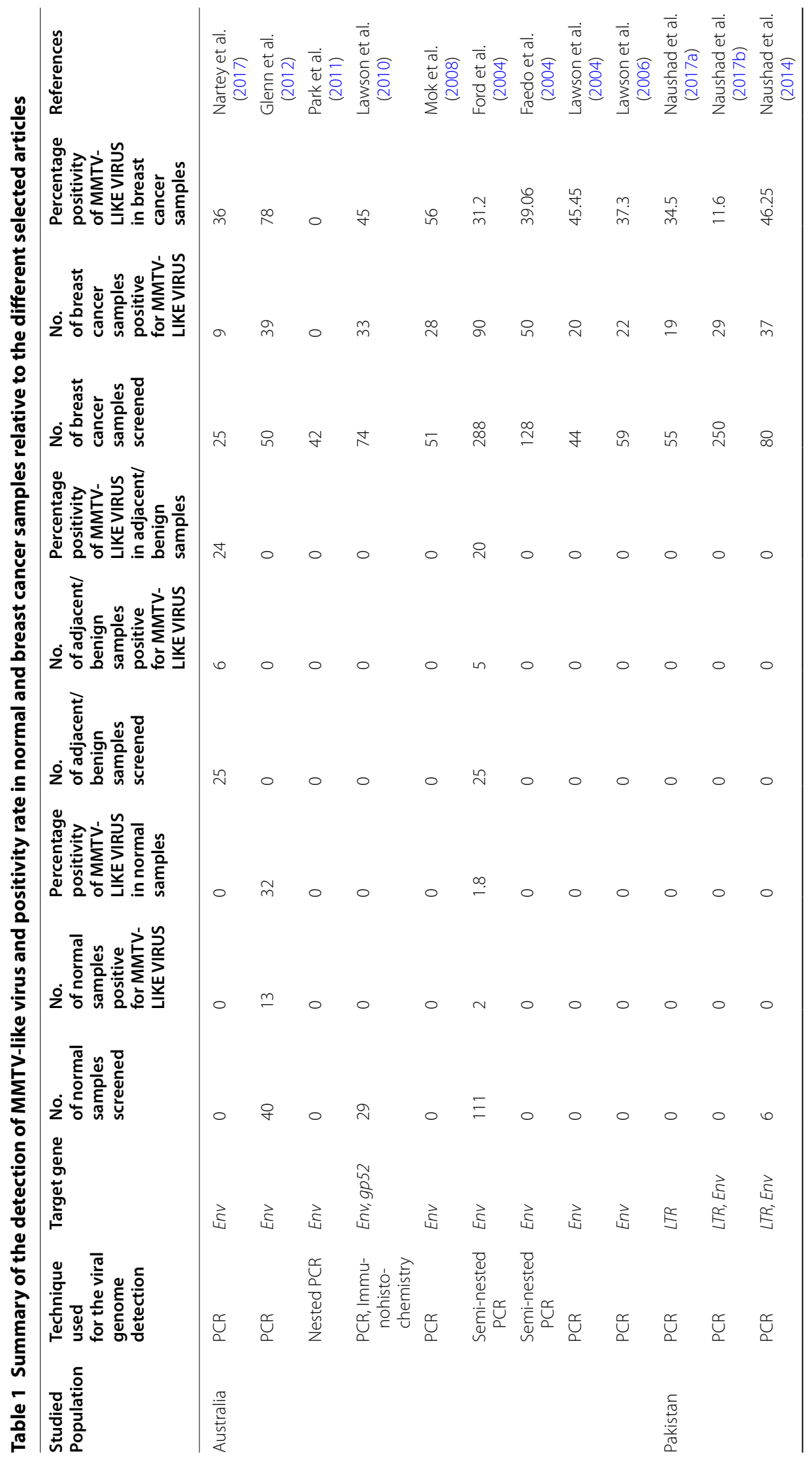









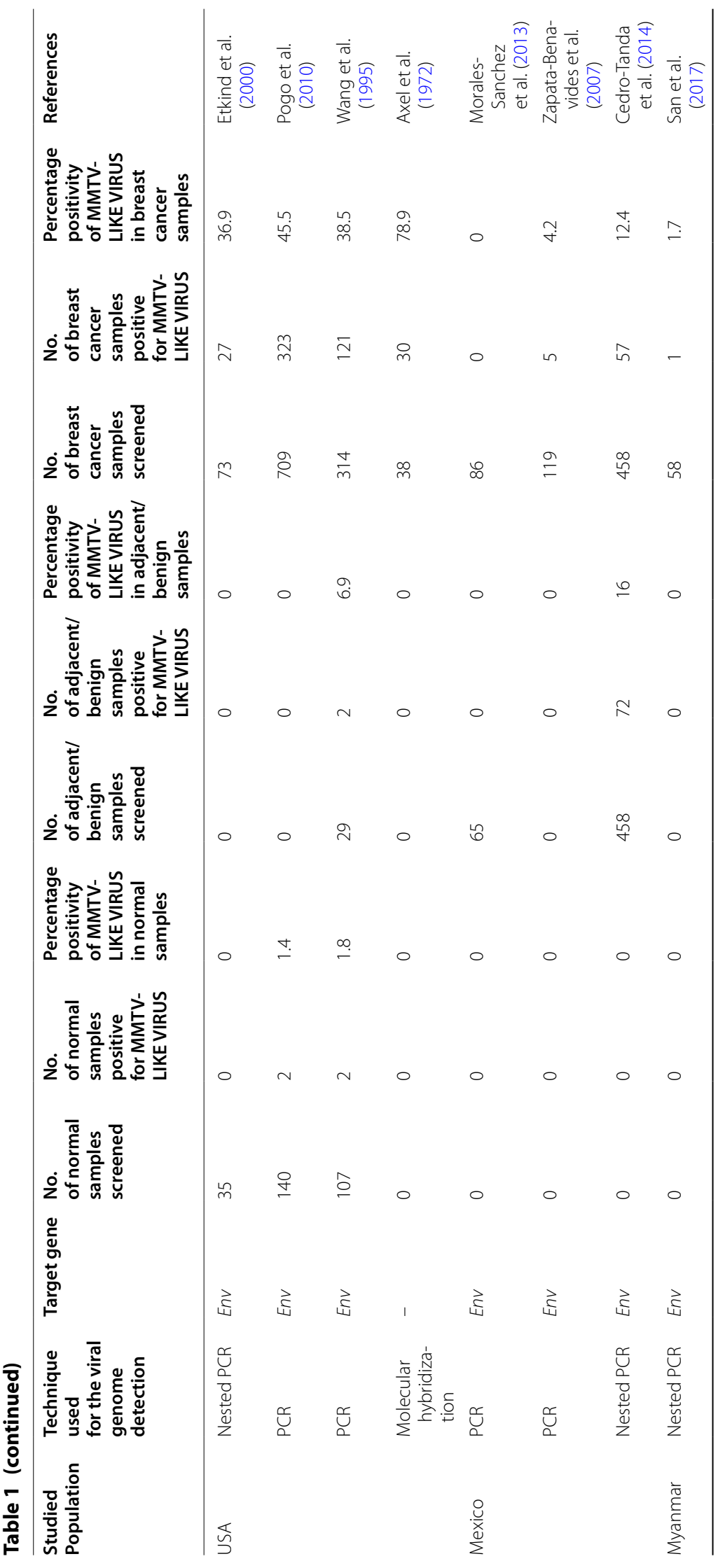




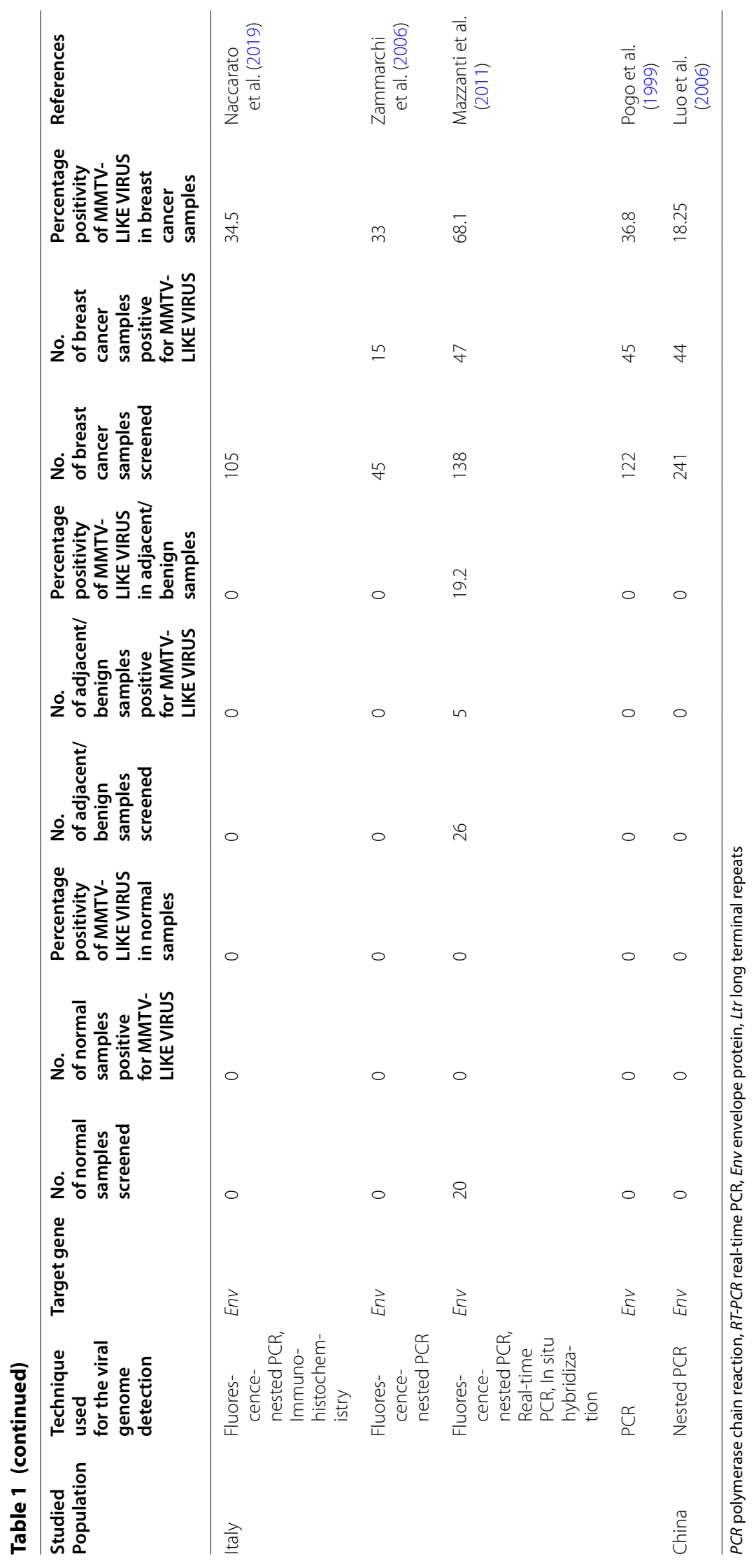




\section{Results}

In total, 40 relevant original articles (Glenn et al. 2012; Axel et al. 1972; Oskouee et al. 2014; Lawson et al. 2006, 2010, 2004; Bindra et al. 2007; Morales-Sanchez et al. 2013; Motamedifar et al. 2012; Park et al. 2011; Tabriz et al. 2013; Witt et al. 2003; Al Dossary et al. 2018; CedroTanda et al. 2014; Etkind et al. 2000; Faedo et al. 2004; Ford et al. 2004, 2003; Hachana et al. 2008; Hafez et al. 2013; Levine et al. 1984; Luo et al. 2006; Mazzanti et al. 2011; Melana et al. 2002, 2001; Mok et al. 2008; Naccarato et al. 2019; Nartey et al. 2017; Naushad et al. 2014, 2017a, b); Pogo et al. 1999, 2010; Reza et al. 2015; San et al. 2017; Shariatpanahi et al. 2017; Slaoui et al. 2014; Wang et al. 1995; Zammarchi et al. 2006; Zapata-Benavides et al. 2007) (Table 1) were found on PubMed which investigated the association of MMTV-like virus with $\mathrm{BC}$ in 24 different populations. Table 1 enlists all these articles with summarized information extracted from these articles including information about the studied population, technique used for the detection of MMTVlike virus, target gene, number (No) of screened samples (normal, adjacent/benign, and $\mathrm{BC}$ ) with their respective identified population-wise positivity ratios. Out of all the 40 studies, in total, 23 studies (Glenn et al. 2012; Oskouee et al. 2014; Bindra et al. 2007; Morales-Sanchez et al. 2013; Motamedifar et al. 2012; Al Dossary et al. 2018; Cedro-Tanda et al. 2014; Etkind et al. 2000; Ford et al. 2004, 2003; Hafez et al. 2013; Lawson et al. 2010; Levine et al. 1984; Mazzanti et al. 2011; Melana et al. 2002, 2001; Nartey et al. 2017; Naushad et al. 2014; Pogo et al. 2010; Reza et al. 2015; Shariatpanahi et al. 2017; Slaoui et al. 2014; Wang et al. 1995) were the case-control studies in which normal, adjacent/benign, and BC samples were screened, while others were not.

The positivity ratio of MMTV-like virus detection in BC samples was varied population-wise from 0\% (Oskouee et al. 2014; Bindra et al. 2007; Morales-Sanchez et al. 2013; Motamedifar et al. 2012; Park et al. 2011; Tabriz et al. 2013; Witt et al. 2003) to 78.9\% (Axel et al. 1972) in all the 40 identified studies, while the positivity ratio of MMTV-like virus detection in normal and adjacent/ benign controls was varied from $0 \%$ (Oskouee et al. 2014; Motamedifar et al. 2012; Al Dossary et al. 2018; Etkind et al. 2000; Ford et al. 2003; Lawson et al. 2010; Mazzanti et al. 2011; Naushad et al. 2014; Reza et al. 2015) to $32 \%$ (Glenn et al. 2012) and 0\% (Bindra et al. 2007; Levine et al. 1984; Melana et al. 2002) to 33.3\% (Slaoui et al. 2014), respectively.

The results obtained after careful evaluation of the extracted data through the Bradford Hill criteria postulates showed that all the identified studies from various populations do not fulfill the major postulates including temporality, consistency, biological gradient, experiment, coherence, specificity, and analogy. Hence, no causal relationship has been suggested between MMTV-like virus and BC patients of any included population.

Polymerase chain reaction (PCR) technique was employed by most of the studies (Glenn et al. 2012; Oskouee et al. 2014; Lawson et al. 2006, 2010, 2004; Bindra et al. 2007; Morales-Sanchez et al. 2013; Motamedifar et al. 2012; Park et al. 2011; Tabriz et al. 2013; Witt et al. 2003; Al Dossary et al. 2018; Cedro-Tanda et al. 2014; Etkind et al. 2000; Faedo et al. 2004; Ford et al. 2004, 2003; Hachana et al. 2008; Hafez et al. 2013; Luo et al. 2006; Mazzanti et al. 2011; Melana et al. 2002, 2001; Mok et al. 2008; Naccarato et al. 2019; Nartey et al. 2017; Naushad et al. 2014, 2017a, b; Pogo et al. 1999, 2010; Reza et al. 2015; San et al. 2017; Shariatpanahi et al. 2017; Slaoui et al. 2014; Wang et al. 1995; Zammarchi et al. 2006; Zapata-Benavides et al. 2007) to detect the presence of MMTV-like virus in the normal, adjacent/benign and BC samples using Env (Glenn et al. 2012; Oskouee et al. 2014; Lawson et al. 2006, 2010, 2004; Bindra et al. 2007; Morales-Sanchez et al. 2013; Motamedifar et al. 2012; Park et al. 2011; Tabriz et al. 2013; Witt et al. 2003; Al Dossary et al. 2018; Cedro-Tanda et al. 2014; Etkind et al. 2000; Faedo et al. 2004; Ford et al. 2004, 2003; Hachana et al. 2008; Hafez et al. 2013; Luo et al. 2006; Mazzanti et al. 2011; Melana et al. 2001; Mok et al. 2008; Naccarato et al. 2019; Nartey et al. 2017; Naushad et al. 2014, 2017a, b; Pogo et al. 1999, 2010; Reza et al. 2015; San et al. 2017; Shariatpanahi et al. 2017; Slaoui et al. 2014; Wang et al. 1995; Zammarchi et al. 2006; Zapata-Benavides et al. 2007), Ltr (Naushad et al. 2014, 2017a, b), and gp52 (Lawson et al. 2010; Levine et al. 1984) gene-specific primers. Additionally, from them, few studies (Glenn et al. 2012; Bindra et al. 2007; Morales-Sanchez et al. 2013; Al Dossary et al. 2018; Cedro-Tanda et al. 2014; Etkind et al. 2000; Ford et al. 2004, 2003; Lawson et al. 2010; Mazzanti et al. 2011; Melana et al. 2001; Mok et al. 2008; Naccarato et al. 2019; Nartey et al. 2017; Naushad et al. 2017; San et al. 2017; Slaoui et al. 2014; Zammarchi et al. 2006; Zapata-Benavides et al. 2007) also employed the second techniques for validating their PCR positive results using second techniques such as immunohistochemistry (Lawson et al. 2010; Naccarato et al. 2019), in situ hybridization (Mazzanti et al. 2011), real-time PCR (Mazzanti et al. 2011), and DNA sequencing analysis (Glenn et al. 2012; Bindra et al. 2007; Morales-Sanchez et al. 2013; Al Dossary et al. 2018; Cedro-Tanda et al. 2014; Etkind et al. 2000; Ford et al. 2004, 2003; Melana et al. 2001; Mok et al. 2008; Nartey et al. 2017; Naushad et al. 2017; San et al. 2017; Slaoui et al. 2014; Zammarchi et al. 2006; ZapataBenavides et al. 2007). On the other hand, few studies also utilized antigen detection method (Levine et al. 
1984) and molecular hybridization method (Axel et al. 1972) for the detection of MMTV-like virus.

Further details regarding population-specific MMTVlike virus etiology in $\mathrm{BC}$ have been given as follows:

In Australia, a total of 09 studies (Glenn et al. 2012; Lawson et al. 2006, 2010, 2004; Park et al. 2011; Faedo et al. 2004; Ford et al. 2004; Mok et al. 2008; Nartey et al. 2017) including 4 case-control studies (Glenn et al. 2012; Ford et al. 2004; Lawson et al. 2010; Nartey et al. 2017) have been reported so far to demonstrate MMTVlike virus etiology in BC. These studies used PCR technique with the help of $E n v$ gene-specific primers for MMTV-LIKE VIRUS detection and documented differential MMTV-like virus detection positivity ratios varying between 0\% (Park et al. 2011) and 78.9\% (Axel et al. 1972) in BC samples, while $20 \%$ (Ford et al. 2004) to $24 \%$ (Nartey et al. 2017) in adjacent/benign samples and $0 \%$ (Lawson et al. 2010) to 32\% (Glenn et al. 2012) in normal controls.

In Pakistan, the association between MMTV-like virus and $\mathrm{BC}$ has been reported in only 03 studies (Naushad et al. 2014, 2017a, b) so far. They utilized PCR technique with primers specifically targeting the Env and LTR regions of the viral genome and documented varying MMTV-like virus detection positivity ratio ranging from $11.6 \%$ (Naushad et al. 2017) to $46.25 \%$ (Naushad et al. 2014) in BC samples.

A total of 05 studies (Oskouee et al. 2014; Motamedifar et al. 2012; Tabriz et al. 2013; Reza et al. 2015; Shariatpanahi et al. 2017) including 04 case-control studies (Oskouee et al. 2014; Motamedifar et al. 2012; Reza et al. 2015; Shariatpanahi et al. 2017) have been carried out in Iran so far, analyzing the association between MMTV-like virus and BC. These studies utilized PCR technique with primers specific for the Env region of the viral genome and documented varying MMTV-like virus detection positivity ratios ranging from 0\% (Oskouee et al. 2014; Motamedifar et al. 2012; Tabriz et al. 2013) to $32.2 \%$ (Shariatpanahi et al. 2017) in BC samples, while $0 \%$ (Oskouee et al. 2014; Motamedifar et al. 2012; Reza et al. 2015) and 5\% (Shariatpanahi et al. 2017) in the normal and adjacent/benign controls, respectively.

A single case-control study (Slaoui et al. 2014) has been conducted in Morocco so far to determine the association between MMTV-like virus and BC. They analyzed $42 \mathrm{BC}$ samples and 18 adjacent/benign controls for the identification of MMTV-like virus in BC using PCR technique with primers specifically hybridizing in the Env sequence of the viral genome. They documented 57.1\% and 33.3\% MMTV-like virus detection positivity ratios in $\mathrm{BC}$ and adjacent/benign controls, respectively.

In Austria, only a single study (Witt et al. 2003) has been reported to date to relating MMTV-like virus presence with $\mathrm{BC}$. They screened $50 \mathrm{BC}$ samples using PCR technique with primers specifically targeting the $E n v$ sequence of the viral genome and they documented $0 \%$ MMTV-like virus detection positivity ratio in $\mathrm{BC}$ samples.

Until now, only a single case-control study (Hafez et al. 2013) has been carried out in Egypt to find out the etiological association between MMTV-like virus and BC. They analyzed $100 \mathrm{BC}$ samples and 50 adjacent/benign controls using PCR technique with Env gene-specific primers for MMTV-like virus detection. They reported the higher MMTV-like virus detection positivity ratio in BC samples (36\%) as compared to the adjacent/benign controls (4\%).

In Vietnam, there has been a single case-control study (Ford et al. 2003) reported so far to find out if MMTVlike virus has any association with BC. They utilized PCR technique with primers specifically targeting the Env sequence of the viral genome and documented a $0.83 \%$ MMTV-like virus detection positivity ratio in BC samples and normal controls, respectively.

Until now, only a single case-control study (Melana et al. 2002) has been carried out in Argentina to elaborate the role of MMTV-like virus in the development of BC. They screened 74 BC samples paired with adjacent/ benign controls using PCR technique with primers targeting the envelope sequence of the viral genome. They documented the higher MMTV-like virus detection positivity ratios in $\mathrm{BC}$ samples (31\%) as compared to the adjacent/benign samples $(0 \%)$ and normal controls $(1.35 \%)$.

In Sweden, a single case-control (Bindra et al. 2007) has been carried out so far to identify the casual relationship between MMTV-like virus and BC using RT-PCR technique and they did not detect MMTV-like virus in any of the $\mathrm{BC}$ or control sample.

So far, a single case-control study (Al Dossary et al. 2018) has been carried out in Saudi Arabia to find out the association between MMTV-like virus and BC. They screened a total of $101 \mathrm{BC}, 51$ normal, and 93 adjacent/ benign controls using PCR technique with primers specific for the Env region of the viral genome and documented MMTV-like virus positivity ratios was higher in the adjacent/benign controls $(9.7 \%)$ as compared to the normal (0\%) and BC samples (5.9\%).

In Tunisia, a total of 03 studies (Hachana et al. 2008; Levine et al. 1984; Pogo et al. 2010) including 02 casecontrol studies (Levine et al. 1984; Pogo et al. 2010) have been reported so far to find out whether MMTVlike virus have any association with BC. They utilized antigen detection method and PCR technique with Env gene-specific primers for MMTV-like virus detection and documented MMTV-like virus detection positivity ratios with varying frequencies ranging from $3.9 \%$ 
(Hachana et al. 2008) to 73.7\% (Pogo et al. 2010) in BC samples, while $0 \%$ (Levine et al. 1984) and 1.4\% (Pogo et al. 2010) in adjacent/benign and normal samples, respectively.

To date, a total of 04 studies (Axel et al. 1972; Etkind et al. 2000; Pogo et al. 2010; Wang et al. 1995) including 3 case-control studies (Etkind et al. 2000; Pogo et al. 2010; Wang et al. 1995) have been carried out in the USA to elaborate the causal association between in adjacent/benign samples and BC. All these studies employed PCR technique with Env gene-specific primers and documented MMTV-like virus detection positivity ratios ranging from $36.9 \%$ (Etkind et al. 2000) to $45.5 \%$ (Pogo et al. 2010) in BC samples, while $6.9 \%$ (Wang et al. 1995) in the adjacent/benign samples and 0\% (Etkind et al. 2000) to 1.8\% (Wang et al. 1995) in the normal controls.

Until now, a total of 03 studies (Morales-Sanchez et al. 2013; Cedro-Tanda et al. 2014; Zapata-Benavides et al. 2007) including two case-control study (MoralesSanchez et al. 2013; Cedro-Tanda et al. 2014) have been reported in Mexico to determine the causal relationship between MMTV-like virus and BC. All these studies utilized PCR with primers specific for the Env gene-specific primers and the documented MMTV-like virus detection positivity ratios varied from 0\% (Morales-Sanchez et al. 2013) to $12.6 \%$ (Cedro-Tanda et al. 2014) in BC samples and $0 \%$ (Morales-Sanchez et al. 2013) to $16 \%$ (CedroTanda et al. 2014) in adjacent/benign controls.

A single study (San et al. 2017) has been conducted in Myanmar to date to relate the MMTV-like virus presence with $\mathrm{BC}$. In total, they analyzed $58 \mathrm{BC}$ samples were using PCR technique with primers specific for the Env region and documented a $1.7 \% 1.7 \%$ detection positivity ratio.

A total of 04 studies (Mazzanti et al. 2011; Naccarato et al. 2019; Pogo et al. 1999; Zammarchi et al. 2006) including a case-control study (Mazzanti et al. 2011) have been carried out in Italy so far, analyzing the etiological association between $1.7 \%$ and BC. These studies utilized the PCR technique with primers specific for the Env region of the viral genome and documented MMTVlike virus detection positivity ratios with varying frequencies ranging from 33\% (Shariatpanahi et al. 2017) to $68.1 \%$ (Mazzanti et al. 2011) in BC samples, while 0\% (Mazzanti et al. 2011) in normal and 19.2\% (Mazzanti et al. 2011) in adjacent/benign controls.

In China, there has been a single study (Luo et al. 2006) reported so far to find out if MMTV-like virus is associated with BC. They utilized PCR technique with primers specifically targeting the $E n v$ sequence of the viral genome and documented $18.25 \%$ MMTV-like virus detection positivity ratio.

\section{Discussion}

$\mathrm{BC}$ is one of the most common types of cancer that infect millions of people worldwide each year. Although recent advancements in the diagnosis and treatment of the $\mathrm{BC}$ have helped to manage the disease but still the prevalence of $\mathrm{BC}$ is on a rise due to unknown underlying mechanisms (Akram et al. 2017).

To date, various individual studies have been carried out worldwide to find the state of association between MMTV-like virus and BC to further uncover the molecular pathways underlying $\mathrm{BC}$ but their results are conflicting. In addition, the statistical meta-analysis was also used by the researchers to analyze the previous individual studies for generating a more meaningful association between MMTV-like virus and BC but due to the shortcomings of statistical meta-analysis, researchers once again failed to establish a more reliable causal relationship between MMTV-like virus and BC.

In the present study, we evaluated the previous studies using a reliable, Bradford Hill criteria to find a causal relationship between MMTV-like virus and BC. In addition, we also evaluated the methodologies used by the previous studies to address the possibility of false-positive and false-negative results for better outcomes.

In total, 40 original articles (Glenn et al. 2012; Axel et al. 1972; Oskouee et al. 2014; Lawson et al. 2006, 2010, 2004; Bindra et al. 2007; Morales-Sanchez et al. 2013; Motamedifar et al. 2012; Park et al. 2011; Tabriz et al. 2013; Witt et al. 2003; Al Dossary et al. 2018; CedroTanda et al. 2014; Etkind et al. 2000; Faedo et al. 2004; Ford et al. 2004, 2003; Hachana et al. 2008; Hafez et al. 2013; Levine et al. 1984; Luo et al. 2006; Mazzanti et al. 2011; Melana et al. 2002, 2001; Mok et al. 2008; Naccarato et al. 2019; Nartey et al. 2017; Naushad et al. 2014, 2017a, b; Pogo et al. 1999, 2010; Reza et al. 2015; San et al. 2017; Shariatpanahi et al. 2017; Slaoui et al. 2014; Wang et al. 1995; Zammarchi et al. 2006; Zapata-Benavides et al. 2007) were included in the present study. The MMTVlike virus positivity ratio reported in these studies was varied between 0\% (Oskouee et al. 2014; Bindra et al. 2007; Morales-Sanchez et al. 2013; Motamedifar et al. 2012; Park et al. 2011; Tabriz et al. 2013; Witt et al. 2003) and 78.9\% (Axel et al. 1972) in BC samples. In most of the case-control studies (Glenn et al. 2012; Oskouee et al. 2014; Bindra et al. 2007; Morales-Sanchez et al. 2013; Motamedifar et al. 2012; Etkind et al. 2000; Ford et al. 2004, 2003; Hafez et al. 2013; Lawson et al. 2010; Levine et al. 1984; Mazzanti et al. 2011; Melana et al. 2002, 2001; Nartey et al. 2017; Naushad et al. 2014; Pogo et al. 2010; Reza et al. 2015; Shariatpanahi et al. 2017; Slaoui et al. 2014; Wang et al. 1995), the positivity ratio for MMTVlike virus detection was higher in the $\mathrm{BC}$ samples as compared to the controls, while in some studies (Al Dossary 
et al. 2018; Cedro-Tanda et al. 2014), MMTV-like virus positivity ratio was higher in the controls as compared to the BC samples. Possible reasons for such populationspecific inequalities in MMTV-like virus detection could be non-modifiable factors such as genetic makeup and socially controllable factors like health-seeking behavior and differential access to the health facilities.

After careful evaluation of the results of identified studies through Bradford hill criteria showed that all the studies failed to fulfill the major postulates including strength, temporality, consistency, biological gradient, experiment, coherence, specificity, and analogy. Hence, we suggested that MMTV-like virus acts as a co-participant in the development of $\mathrm{BC}$ rather than having a causal relationship that might combine with the other viruses and such as human immunodeficiency virus (HIV) and hepatitis $\mathrm{C}$ virus (HCV), other factors including genetic abnormalities, smoking, alcohol consumption, and obesity may increase a person's risk of developing $\mathrm{BC}$ by affecting the body's immune system.

However, limitations and some of the major issues related with methodologies used in the included studies have been discussed below.

\section{Possible causes of false-negative results}

In few studies, the presence of MMTV-like virus was not detected in any of the analyzed samples. How we can be sure that their negative results of MMTV-like virus detection were not due to the poor quality of the extracted DNA? To overcome this issue, most of the studies (Glenn et al. 2012; Oskouee et al. 2014; Lawson et al. 2006, 2010, 2004; Bindra et al. 2007; MoralesSanchez et al. 2013; Motamedifar et al. 2012; Park et al. 2011; Tabriz et al. 2013; Al Dossary et al. 2018; CedroTanda et al. 2014; Etkind et al. 2000; Faedo et al. 2004; Ford et al. 2004, 2003; Hachana et al. 2008; Hafez et al. 2013; Levine et al. 1984; Luo et al. 2006; Melana et al. 2002, 2001; Mok et al. 2008; Naccarato et al. 2019; Nartey et al. 2017; Naushad et al. 2014, 2017a, b; Pogo et al. 1999, 2010; Reza et al. 2015; San et al. 2017; Shariatpanahi et al. 2017; Slaoui et al. 2014; Wang et al. 1995; Zammarchi et al. 2006; Zapata-Benavides et al. 2007) used internal or positive-control but two studies (Witt et al. 2003; Mazzanti et al. 2011) did not use positive control so there is no way to confirm their negative results. Moreover, the risk of contamination during PCR can never be ruled out completely and it could lead to false-negative results. In total, two studies (Mazzanti et al. 2011; Naccarato et al. 2019) prepared histological specimens using laser microdissection approach during which morphology as well as the quality of the cellular material can be compromised (Chung and Shen 2015). So, these could be possible reasons behind the completely negative results of these studies (Oskouee et al. 2014; Bindra et al. 2007; MoralesSanchez et al. 2013; Motamedifar et al. 2012; Park et al. 2011; Tabriz et al. 2013; Witt et al. 2003).

\section{Possible causes of false-positive results}

Few studies (Oskouee et al. 2014; Bindra et al. 2007; Motamedifar et al. 2012; Park et al. 2011; Witt et al. 2003; Cedro-Tanda et al. 2014; Faedo et al. 2004; Ford et al. 2004; Hachana et al. 2008; Hafez et al. 2013; Luo et al. 2006; Mazzanti et al. 2011; Naccarato et al. 2019; San et al. 2017; Shariatpanahi et al. 2017; Zammarchi et al. 2006) used nested PCR for the detection of MMTV-like virus, a method that is more prone to the contamination and could lead to false-positive results. Although, these studies cited a good laboratory practices but the chances of contamination during PCR can never be ruled out completely.

Almost all the studies (Glenn et al. 2012; Oskouee et al. 2014; Lawson et al. 2006, 2010, 2004; Bindra et al. 2007; Morales-Sanchez et al. 2013; Motamedifar et al. 2012; Park et al. 2011; Tabriz et al. 2013; Witt et al. 2003; Al Dossary et al. 2018; Cedro-Tanda et al. 2014; Etkind et al. 2000; Faedo et al. 2004; Ford et al. 2004, 2003; Hachana et al. 2008; Hafez et al. 2013; Luo et al. 2006; Mazzanti et al. 2011; Melana et al. 2002, 2001; Mok et al. 2008; Naccarato et al. 2019; Nartey et al. 2017; Naushad et al. 2014, 2017a, b; Pogo et al. 1999, 2010; Reza et al. 2015; San et al. 2017; Shariatpanahi et al. 2017; Slaoui et al. 2014; Wang et al. 1995; Zammarchi et al. 2006; Zapata-Benavides et al. 2007) used PCR technique for the initial detection of MMTV-like virus but only few studies (Glenn et al. 2012; Bindra et al. 2007; Morales-Sanchez et al. 2013; Al Dossary et al. 2018; Cedro-Tanda et al. 2014; Etkind et al. 2000; Ford et al. 2004, 2003; Lawson et al. 2010; Mazzanti et al. 2011; Melana et al. 2001; Mok et al. 2008; Naccarato et al. 2019; Nartey et al. 2017; Naushad et al. 2017; San et al. 2017; Slaoui et al. 2014; Zammarchi et al. 2006; Zapata-Benavides et al. 2007) validated their PCR positive results using second techniques such as immunohistochemistry (Lawson et al. 2010; Naccarato et al. 2019), In situ hybridization (Mazzanti et al. 2011), realtime PCR (Mazzanti et al. 2011), and DNA sequencing analysis (Glenn et al. 2012; Bindra et al. 2007; MoralesSanchez et al. 2013; Al Dossary et al. 2018; Cedro-Tanda et al. 2014; Etkind et al. 2000; Ford et al. 2004, 2003; Melana et al. 2001; Mok et al. 2008; Nartey et al. 2017a, b; San et al. 2017; Slaoui et al. 2014; Zammarchi et al. 2006; Zapata-Benavides et al. 2007). Expression of PgR, HER2, Wnt-1, laminin receptor could be used as surrogate biomarker in MMTV-like virus-infected BC patients. Along with MMTV-like virus detection, these surrogate biomarkers were also analyzed by some studies (Faedo et al. 2004; Hachana et al. 2008; Lawson et al. 2010, 2004; Pogo 
et al. 1999; Reza et al. 2015) to further validate their findings, out of which (Faedo et al. 2004; Lawson et al. 2010, 2004; Pogo et al. 1999; Reza et al. 2015) have validated their findings by analyzing these surrogate biomarkers, while the other studies (Hachana et al. 2008; Pogo et al. 1999) were failed to validate their findings through surrogate biomarkers. Such deviations in the results of previous studies raise a big question mark on the selection of appropriate technique and their sensitivities.

\section{Comparison of normal, benign, and malignant samples}

Case-control studies are necessary to establish a causal relationship between the causative agent and the disease. Some of the studies we summarized analyzed only the BC samples (Axel et al. 1972; Lawson et al. 2006, 2004; Park et al. 2011; Tabriz et al. 2013; Witt et al. 2003; Faedo et al. 2004; Hachana et al. 2008; Luo et al. 2006; Mok et al. 2008; Naccarato et al. 2019; Naushad et al. 2014, 2017; Pogo et al. 1999; San et al. 2017; Zammarchi et al. 2006; Zapata-Benavides et al. 2007) and did not allow us to compare their results with normal or adjacent/benign controls. However, most of the studies (Glenn et al. 2012; Oskouee et al. 2014; Bindra et al. 2007; Morales-Sanchez et al. 2013; Motamedifar et al. 2012; Al Dossary et al. 2018; Cedro-Tanda et al. 2014; Etkind et al. 2000; Ford et al. 2004, 2003; Hafez et al. 2013; Lawson et al. 2010; Levine et al. 1984; Mazzanti et al. 2011; Melana et al. 2002, 2001; Nartey et al. 2017; Naushad et al. 2014; Pogo et al. 2010; Reza et al. 2015; Shariatpanahi et al. 2017; Slaoui et al. 2014; Wang et al. 1995) also analyzed the normal and adjacent/benign tissues along with $\mathrm{BC}$ samples and comparison of their results demonstrated that MMTV-like virus detection positivity ratios in BC samples were higher in Glenn et al. (2012), Oskouee et al. (2014), Bindra et al. (2007), Morales-Sanchez et al. (2013), Motamedifar et al. (2012), Etkind et al. (2000), Ford et al. (2004, 2003), Hafez et al. (2013), Lawson et al. (2010), Levine et al. (1984), Mazzanti et al. (2011), Melana et al. (2002, 2001), Nartey et al. (2017), Naushad et al. (2014), Pogo et al. (2010), Reza et al. (2015), Shariatpanahi et al. (2017), Slaoui et al. (2014), and Wang et al. (1995) studies, while lower in Al Dossary et al. (2018) and CedroTanda et al. (2014) studies as compared to the normal and adjacent/benign controls. However, none of the studies has reported the association of MMTV-like virus with specific $\mathrm{BC}$ subtype and histologic grade.

\section{Conclusion}

The results of this comprehensive review are controversial. They failed to prove the causal relationship between MMTV-like virus and $\mathrm{BC}$ rather suggested it as co-participant in the pathogenesis of BC. However, due to limitations of the methodologies used by the previous studies to detect the presence of MMTV-like virus in BC, additional experiments are required to prove the MMTV-like virus etiology in $\mathrm{BC}$.

\section{Abbreviations}

DNA: Deoxyribonucleic acid; Env: Envelope gene; EBV: Epstein-Barr virus; HPV: Human papilloma virus; Ltr: Long terminal repeats; MeSH: Medical subject headings; MMTV: Mouse mammary tumor virus; PCR: Polymerase chain reaction; RT-PCR: Real-time polymerase chain reaction; RNA: Ribonucleic acid.

\section{Acknowledgements}

Not applicable.

\section{Authors' contributions}

YH formulated the idea; $\mathrm{MU}$ collected the data and performed the statistical analysis with the help of MA. MU wrote the first draft of the manuscript which is finalized by $\mathrm{YH}$. All authors read and approved the final manuscript.

\section{Funding}

Not applicable.

\section{Availability of data and materials \\ Not applicable.}

Ethics approval and consent to participate Not applicable.

\section{Consent for publication}

Not applicable.

\section{Competing interests}

The authors declare that they have no competing interests.

Received: 13 February 2020 Accepted: 22 October 2020

Published online: 31 October 2020

\section{References}

Akram M, Iqbal M, Daniyal M, Khan AU (2017) Awareness and current knowledge of breast cancer. Biol Res 1:33-33

Al Dossary R, Alkharsah KR, Kussaibi H (2018) Prevalence of Mouse Mammary Tumor Virus (MMTV)-like sequences in human breast cancer tissues and adjacent normal breast tissues in Saudi Arabia. BMC Cancer 1:170

Axel R, Schlom J, Spiegelman S (1972) Presence in human breast cancer of RNA homologous to mouse mammary tumour virus RNA. Nature 5332:32-36

Bindra A, Muradrasoli S, Kisekka R, Nordgren H, Wärnberg F, Blomberg J (2007) Search for DNA of exogenous mouse mammary tumor virus-related virus in human breast cancer samples. J Gen Virol 6:1806-1809

Cedro-Tanda A, Córdova-Solis A, Juárez-Cedillo T, Pina-Jiménez E, HernándezCaballero ME, Moctezuma-Meza C et al (2014) Prevalence of HMTV in breast carcinomas and unaffected tissue from Mexican women. BMC Cancer 1:942

Chung SH, Shen W (2015) Laser capture microdissection: from its principle to applications in research on neurodegeneration. Neural Regen Res 6:897-898

Coleman MP, Quaresma M, Berrino F, Lutz JM, De Angelis R, Capocaccia R et al (2008) Cancer survival in five continents: a worldwide population-based study (CONCORD). Lancet Oncol 8:730-756

Etkind P, Du J, Khan A, Pillitteri J, Wiernik PH (2000) Mouse mammary tumor virus-like ENV gene sequences in human breast tumors and in a lymphoma of a breast cancer patient. Clin Cancer Res 4:1273-1278

Faedo M, Ford CE, Mehta R, Blazek K, Rawlinson WD (2004) Mouse mammary tumor-like virus is associated with p53 nuclear accumulation and progesterone receptor positivity but not estrogen positivity in human female breast cancer. Clin Cancer Res 13:4417-4419 
Fedak KM, Bernal A, Capshaw ZA, Gross S (2015) Applying the Bradford Hill criteria in the 21st century: how data integration has changed causal inference in molecular epidemiology. Emerg Themes Epidemiol. 12:14

Ford CE, Faedo M, Crouch R, Lawson JS, Rawlinson WD (2004) Progression from normal breast pathology to breast cancer is associated with increasing prevalence of mouse mammary tumor virus-like sequences in men and women. Cancer Res 14:4755-4759

Ford CE, Tran D, Deng Y, Rawlinson WD, Lawson JS (2003) Mouse mammary tumor virus-like gene sequences in breast tumors of Australian and Vietnamese women. Clin Cancer Res 3:1118-1120

Glenn WK, Whitaker NJ, Lawson JS (2012) High risk human papillomavirus and Epstein Barr virus in human breast milk. BMC Res Notes 1:1-4

Hachana M, Trimeche M, Ziadi S, Amara K, Gaddas N, Mokni M et al (2008) Prevalence and characteristics of the MMTV-like associated breast carcinomas in Tunisia. Cancer Lett 2:222-230

Hafez MM, Hassan ZK, Kamel MM, Elrouby MN, Zekri ARN (2013) Expression of MMTV-like env gene in Egyptian Breast Cancer Patients. Egypt J Med Microbiol 1233:1-7

Jemal A, Bray F, Center MM, Ferlay J, Ward E, Forman D (2011) Global cancer statistics. CA Cancer J Clin 2:69-90

Lawson JS (2009) Do viruses cause breast cancer? Methods Mol Biol 471:421-438

Lawson JS, Glenn WK, Salmons B, Ye Y, Heng B, Moody P et al (2010) Mouse mammary tumor virus-like sequences in human breast cancer. Cancer Res 9:3576-3585

Lawson JS, Tran DD, Carpenter E, Ford CE, Rawlinson WD, Whitaker NJ et al (2006) Presence of mouse mammary tumour-like virus gene sequences may be associated with morphology of specific human breast cancer. J Clin Pathol 12:1287-1292

Lawson JS, Tran DD, Ford C, Rawlinson WD (2004) Elevated expression of the tumor suppressing protein p53 is associated with the presence of mouse mammary tumor-like env gene sequences (MMTV-like) in human breast cancer. Breast Cancer Res Treat 1:13-17

Levine PH, Mesa-Tejada R, Keydar I, Tabbane F, Spiegelman S, Mourali N (1984) Increased incidence of mouse mammary tumor virus-related antigen in Tunisian patients with breast cancer. Int J Cancer 3:305-308

Luo T, Wu X-T, Zhang M-M, Qian K. Study of mouse mammary tumor virus-like gene sequences expressing in breast tumors of Chinese women. Sichuan da xue xue bao Yi xue ban J Sichuan Univ Med Sci Ed 2006;6:844-846, 851.

Mazzanti CM, Al Hamad M, Fanelli G, Scatena C, Zammarchi F, Zavaglia K et al (2011) A mouse mammary tumor virus env-like exogenous sequence is strictly related to progression of human sporadic breast carcinoma. Am J Pathol 4:2083-2090

Melana SM, Holland JF, Pogo BG (2001) Search for mouse mammary tumor virus-like env sequences in cancer and normal breast from the same individuals. Clin Cancer Res 2:283-284

Melana S, Picconi M, Rossi C, Mural J, Alonio L, Teyssie A et al (2002) Detection of murine mammary tumor virus (MMTV) env gene-like sequences in breast cancer from Argentine patients. Medicina 4:323-327

Mok MT, Lawson JS, lacopetta BJ, Whitaker NJ (2008) Mouse mammary tumor virus-like env sequences in human breast cancer. Int J Cancer 12:2864-2870

Morales-Sanchez A, Molina-Munoz T, Martinez-Lopez JL, Hernandez-Sancen P, Mantilla A, Leal YA et al (2013) No association between Epstein-Barr Virus and Mouse Mammary Tumor Virus with breast cancer in Mexican women. Sci Rep 3:2970

Motamedifar M, Saki M, Ghaderi A (2012) Lack of association of mouse mammary tumor virus-like sequences in Iranian breast cancer patients. Med Princ Pract Int J Kuwait Univ Health Sci Cent 3:244-248

Naccarato AG, Lessi F, Zavaglia K, Scatena C, Al Hamad MA, Aretini P et al (2019) Mouse mammary tumor virus (MMTV)-like exogenous sequences are associated with sporadic but not hereditary human breast carcinoma. Aging (Albany NY) 17:7236-7241

Nartey T, Mazzanti CM, Melana S, Glenn WK, Bevilacqua G, Holland JF et al (2017) Mouse mammary tumor-like virus (MMTV) is present in human breast tissue before development of virally associated breast cancer. Infect Agent Cancer 1:1
Naushad W, Bin Rahat T, Gomez MK, Ashiq MT, Younas M, Sadia H (2014) Detection and identification of mouse mammary tumor virus-like DNA sequences in blood and breast tissues of breast cancer patients. Tumour Biol J Int Soc Oncodev Biol Med 8:8077-8086

Naushad W, Surriya O, Sadia H (2017a) Prevalence of EBV, HPV and MMTV in Pakistani breast cancer patients: a possible etiological role of viruses in breast cancer. J Mol Epidemiol Evol Genet Infect Dis 54:230-237

Naushad W, Ayub S, Sadia H (2017b) Significant correlation of MMTV (Mouse mammary tumor virus) LTR gene with hormone receptor status in peripheral blood samples of breast cancer patients from North Pakistan.

Oskouee MA, Shahmahmoodi S, Jalilvand S, Mahmoodi M, Ziaee A-A, Esmaeili HA et al (2014) No evidence of mammary tumor virus env gene-like sequences among Iranian women with breast cancer. Intervirology 6:353-356

Park DJ, Southey MC, Giles GG, Hopper JL (2011) No evidence of MMTV-like env sequences in specimens from the Australian Breast Cancer Family Study. Breast Cancer Res Treat 1:229-235

Pogo BGT, Holland JF, Levine PH (2010) Human mammary tumor virus in inflammatory breast cancer. Cancer S11:2741-2744

Pogo BG, Melana SM, Holland JF, Mandeli JF, Pilotti S, Casalini P et al (1999) Sequences homologous to the mouse mammary tumor virus env gene in human breast carcinoma correlate with overexpression of laminin receptor. Clin Cancer Res 8:2108-2111

Reza MA, Reza MH, Mahdiyeh L, Mehdi F, Hamid ZN (2015) Evaluation frequency of Merkel cell polyoma, Epstein-Barr and mouse mammary tumor viruses in patients with breast cancer in Kerman, Southeast of Iran. Asian Pac J Cancer Prev APJCP 16:7351-7357

San TH, Fujisawa M, Fushimi S, Yoshimura T, Ohara T, Soe L et al (2017) Low prevalence of human mammary tumor virus (HMTV) in breast cancer patients from Myanmar. Infect Agent Cancer 12:20-20

Shariatpanahi S, Farahani N, Salehi AR, Salehi R (2017) High prevalence of mouse mammary tumor virus-like gene sequences in breast cancer samples of Iranian women. Nucleosides Nucleotides Nucleic Acids 10:621-630

Sharma GN, Dave R, Sanadya J, Sharma P, Sharma KK (2010) Various types and management of breast cancer: an overview. J Adv Pharm Technol Res 2:109-126

Slaoui M, El Mzibri M, Razine R, Qmichou Z, Attaleb M, Amrani M (2014) Detection of MMTV-Like sequences in Moroccan breast cancer cases. Infect Agent Cancer 1:37

Tabriz HM, Zendehdel K, Shahsiah R, Fereidooni F, Mehdipour B, Hosseini ZM (2013) Lack of detection of the mouse mammary tumor-like virus (MMTV) Env gene in Iranian women breast cancer using real time PCR. Asian Pac J Cancer Prev APJCP 5:2945-2948

Wang Y, Holland JF, Bleiweiss IJ, Melana S, Liu X, Pelisson I et al (1995) Detection of mammary tumor virus env gene-like sequences in human breast cancer. Cancer Res 22:5173-5179

Wang F, Hou J, Shen Q, Yue Y, Xie F, Wang X et al (2014) Mouse mammary tumor virus-like virus infection and the risk of human breast cancer: a meta-analysis. Am J Transl Res 3:248-266

Witt A, Hartmann B, Marton E, Zeillinger R, Schreiber M, Kubista E (2003) The mouse mammary tumor virus-like env gene sequence is not detectable in breast cancer tissue of Austrian patients. Oncol Rep 4:1025-1029

Zammarchi F, Pistello M, Piersigilli A, Murr R, Di Cristofano C, Naccarato AG et al (2006) MMTV-like sequences in human breast cancer: a fluorescent PCR/ laser microdissection approach. J Pathol 4:436-444

Zapata-Benavides P, Saavedra-Alonso S, Zamora-Avila D, Vargas-Rodarte C, Barrera-Rodríguez R, Salinas-Silva J et al (2007) Mouse mammary tumor virus-like gene sequences in breast cancer samples of Mexican women. Intervirology 6:402-407

Zucca-Matthes G, Urban C, Vallejo A (2016) Anatomy of the nipple and breast ducts. Gland Surg 1:32-36

\section{Publisher's Note}

Springer Nature remains neutral with regard to jurisdictional claims in published maps and institutional affiliations. 